Arqueología y Sociedad,

№ 21, 2010

\title{
Perspectiva arqueOlógica en la eCONOMía INCAICA ${ }^{1}$
}

\section{Craig Morris \\ Julián Idilio Santillana}

Es evidente que para una reconstrucción válida de las instituciones andinas se necesitan diversas estrategias que dispongan de distintos tipos de información. Las evidencias etnográficas, documentales y arqueológicas son incompletas por separado y es preciso enlazadas de manera sistemática ${ }^{2}$. A pesar de lo obvio del principio, en la práctica ha sido muy difícil de aplicar. En particular, se ha dejado de integrar a la arqueología en, los estudios vinculados a asuntos institucionales y de organización. Aunque Menzel, en su artículo sobre la dominación incaica de la costa sur del Perú (1959), ha mostrado lo que es posible lograr cuando se aplica la arqueología a estos problemas, solo ahora estamos en condiciones de esperar que la arqueología aporte algo más de lo que tradicionalmente le ha interesado, o sea el estudio de la tecnología, las subsistencias y la cronología.

No creo que sea posible, indicar paso a paso una metodología básica que describa cómo se ha de combinar exitosamente la arqueología con la etnohistoria y otras técnicas. Todo procedimiento varía en gran medida debido a las diferencias entre los temas mismos, las características de los investigadores y las informaciones disponibles. No obstante, hay dos puntos que merecen un énfasis especial por ser de particular importancia: en primer lugar, es necesario hacer un esfuerzo constante para eliminar las fronteras disciplinarias. y sub-disciplinarias, pues es posible que las soluciones a interrogantes planteados por una subdisciplina puedan ser abordadas con los métodos y los datos manejados por otra; en segundo lugar, las investigaciones deben ser diseñadas, desde su inicio, de tal manera que incluyan estrategias múltiples anticipando cómo las diversas técnicas se verifican entre sí y como todas pueden contribuir, a un conjunto uniforme de objetivos.

Este informe es un ejemplo de cómo la arqueología puede proporcionar nuevas informaciones sobre diversos y complejos problemas vinculados al estudio del estado incaico. Aunque las conclusiones de la investigación todavía están en una fase preliminar, es de esperar que muestren algo sobre la manera de enfrentar los problemas cuya importancia ya se ha establecido a través de

1 Parte de los materiales de este estudio fue utilizado por los autores en una ponencia al I Congreso Peruano del Hombre y la Cultura: Andina y por Morris en "Reconstructing Patterns of Non-Agricultural Production in the lnca Economy: Archaeology and Documents in Instituttonal Analysis", in Charlotte Móore (editor) Reconstructing of Complex Societies, The American Schools of Oriental Research, Cambridge 1974.

2 Las obras siguientes son ejemplos de investigaciones básicas, las cuales, consciente y eficazmente, combinan documentos y arqueología en la reconstrucción de ciertos aspectos de sociedades e instituciones sociales. No se espera que la lista sea exhaustiva, sino que provea de una introducción aparte del trabajo que se está llevando a cabo: Adams (1965: 1972); Calnek (1972); Charlton (1969); Rowe ( 1967); Rostworowski (1977, caps: 1, 3, 5 y 6 ).

Nota. La reedición de este artículo fue autorizado por la dirección de la Revista Histórica, PUCP, 2010. En la publicación original de 1978 no aparecen las Figuras 1 y 2. Las versiones finales de ambas figuras fueron publicadas en diversas ocasiones; y las que se publican en esta reedición han sido tomadas del libro Huánuco Pampa. An Inca City and its Hinterland by Craig Morris and Donald E. Thompson. 1985. New Aspects of Antiquity. Generald Editor Colin Renfrew. Thames and Hudson Ltd, London. "Reconstructing Patterns of Non-Agricultural Production in the lnca Economy: Archaeology and Documents in Instituttonal Analysis”, en Charlotte Móore (editor) Reconstructing of Complex Societies, The American Schools of Oriental Research, Cambridge 1974. 
los estudios etnohistóricos. Además, se espera que el éxito de aquellos estudios sea tal que se alentará la realización de otras investigaciones semejantes, las cuales abordarían una gran variedad de temas esenciales para la comprensión de las instituciones andinas.

Es evidente que para una reconstrucción válida de las instituciones andinas se necesitan diversas estrategias que dispongan de distintos tipos de información. Las evidencias etnográficas, documentales y arqueológicas son incompletas por separado y es preciso enlazarlas de manera sistemática ${ }^{3}$. A pesar de lo obvio del principio, en la práctica ha sido muy difícil de aplicar. En particular, se ha dejado de integrar a la arqueología en los estudios vinculados a asuntos institucionales y de organización. Aunque Menzel, en su artículo sobre la dominación incaica de la costa sur del Perú (1959), ha mostrado lo que es posible lograr cuando se aplica la arqueología a estos problemas, solo ahora estamos en condiciones de esperar que la Arqueología aporte algo más de lo que tradicionalmente le ha interesado, o sea el estudio de la tecnología, las subsistencias y la cronología.

No creo que sea posible, indicar paso a paso una metodología básica que describa cómo se ha de combinar exitosamente la arqueología con la etnohistoria y otras técnicas. Todo procedimiento varía en gran medida debido a las diferencias entre los temas mismos, las características de los investigadores y las informaciones .disponibles. No obstante, hay dos puntos que merecen un énfasis especial por ser de particular importancia: en primer lugar, es necesario hacer un esfuerzo constante para eliminar las fronteras disciplinarias. y sub-disciplinarias, pues es posible que las soluciones a interrogantes planteados por una subdisciplina puedan ser abordadas

3 Las obras siguientes son ejemplos de investigaciones básicas, las cuales, consciente y eficazmente, combinan documentos y arqueología en la reconstrucción de ciertos aspectos de sociedades e instituciones sociales. No se espera que la lista sea exhaustiva, sino que provea de una introducción aparte del trabajo que se está llevando a cabo: Adams (1965: 1972); Calnek (1972); Charlton (1969); Rowe ( 1967) ; Rostworowski (1977, caps: $1,3,5$ y 6$)$. con los métodos y los datos manejados por otra; en segundo lugar, las investigaciones deben ser diseñadas, desde su inicio, de tal manera que incluyan estrategias múltiples anticipando cómo las diversas técnicas se verifican entre sí y como todas pueden contribuir, a un conjunto uniforme de objetivos.

Este informe es un ejemplo de cómo la arqueología puede proporcionar nuevas informaciones sobre diversos y complejos problemas vinculados al estudio del estado incaico. Aunque las conclusiones de la investigación todavía están en una fase preliminar, es de esperar que muestren algo sobre la manera de enfrentar los problemas cuya importancia ya se ha establecido a través de los estudios etnohistóricos. Además, se espera que el éxito de aquellos estudios sea tal que se alentará la realización de otras investigaciones semejantes, las cuales abordarían una gran variedad de temas esenciales para la comprensión de las instituciones andinas.

\section{INVESTIGACIONES ARQUEOLÓGICO-DOCUMENTALES EN HUÁNUCO}

En 1963 se inició en el departamento de Huánuco, en la sierra central peruana, un estudio que combinaba técnicas arqueológicas e históricas en la investigación multidisciplinaria conocida como "Un Estudio de la Vida Provincial Incaica" y dirigida por John V. Murra. Participaron en este programa de investigaciones basadas sobre fuentes documentales específicas y excepcionalmente productivas (Ortiz de Zúñiga [1562], 1967, 1972), arqueólogos e historiadores, así como etnólogos y un botánico. El equipo concentró su atención en varios grupos locales integrados al estado incaico así como en el camino incaico, incluyendo los centros de poder estatal allí localizados; también se vieron las relaciones entre ambos factores. La investigación actual se deriva directamente de aquel estudio, concentrándose en los aspectos urbanos de la dominación incaica en las regiones provinciales. ${ }^{4}$

4 El proyecto del Dr. Murra, en el cual Morris colaboró como auxiliar, fue subvencionado por la National 
Como en el espacio disponible no se puede analizar toda la investigación en Huánuco, se considerará solo un rasgo del patrón incaico -la producción no agrícola- para mostrar como se ha tratado de diseñar las investigaciones arqueológicas utilizando como base la documentación. Y porque la mayoría de los datos nuevos están relacionados con la manufactura de tejidos, éste será el tema principal que nos ocupará.

La producción especializada no agrícola siempre ha sido una parte integral de las consideraciones sobre el desarrollo y la naturaleza de las civilizaciones tempranas. Los arqueólogos se han interesado especialmente en la formación de especialistas en esta época y en los avances tecnológicos que se lograron. Pero el material ordinariamente tratado no ha servido mucho para el análisis de otros problemas y ramificaciones de la producción no agrícola y artesanal, que también son cruciales para comprender las sociedades en cuestión. Un enfoque sistemático que combine el uso de materiales documentales con lo arqueológico, cuando se disponga de ambos, producirá un panorama más completo. Para poder obtener esta mejor perspectiva, debemos responder a tres conjuntos generales de preguntas.

El primer grupo tiene un carácter mayormente tecnológico: ¿Cómo se manufacturaban estos productos? ¿Cuáles eran los materiales utilizados y dónde se originaban? Las técnicas de producción iacentuaban la cantidad o la calidad? ¿Había claras distinciones en la calidad de los productos? Estas preguntas se pueden resolver casi siempre por medio de la arqueología, excepto en el caso de productos como los tejidos que ordinariamente no se conservan. El material histórico tiene importancia más marginal, aunque puede ser útil para indicar el origen de

Science Foundation, proyecto GS 42. Antes del inicio del proyecto, Murra (1962b) escribió un informe que examinó algunos de los problemas tratados. Se ha publicado muchos de los resultados en ensayos que acompañan los documentos que se usaron para organizar el trabajo (Ortiz 1562; 1967; 1972). Véase también Thompson (1968a; 1968b; 1969), Morris y Thompson (1970), y Morris (1966; 1967; 1971; 1972a). los materiales y para señalar los antiguos puntos de vista sobre las diferencias en la calidad de los productos y sobre la clasificación de los objetos en general.

El segundo conjunto de preguntas trata de la organización: ¿Se organizaba acaso a los productores en unidades específicas de producción? ¿Trabajaban a tiempo completo, o solo por horas? ¿Cuál era su posición social y económica? ¿Cómo se administraba la producción? Es posible reunir datos arqueológicos relativos a todas estas preguntas, pero a la interpretación es difícil, lográndose mejor cuando analiza otra serie de hipótesis alternativas y más específicas. Estas hipótesis se formulan con mayor facilidad con la ayuda de documentos.

Finalmente, aparecen preguntas más amplias que consideran no solo los contenidos socioeconómicos, sino también los contextos políticos e ideológicos relacionados con los bienes y su producción: ¿Cómo se distribuían estos bienes? ¿Se limitaba el uso de ciertos bienes a clases o contextos sociales específicos? ¿En qué se fundaba la demanda de los diversos productos? ¿Iba más allá de una utilidad básica? Estas preguntas son las más difíciles, pero tal vez las más importantes, si el estudio de los artefactos y su producción ha de contribuir a la reconstrucción y a la comprensión de la sociedad en su totalidad. Es aquí también donde se precisa coordinar estrechamente los estudios documentales y la arqueología. El arqueólogo en particular, tiene ventajas cuando dispone de uno o varios modelos de la sociedad y sus instituciones, que posibilitan así la formulación de interrogantes en esos tejidos. Como dichos modelos a menudo son incompletos o no están adecuadamente comprobados, la arqueología puede aportar nuevas informaciones vitales.

Mucho del material etnohistórico apropiado al estudio de los productos incaicos no agrícolas ha sido tratado por Rowe (f946) y por Murra (1956 y 1975) en obras generales sobre la cultura y la economía incaicas. Además, el artículo de Murra, "La función del tejido en varios con- 
textos sociales del estado Inca" ([1958] 1975), nos ofrece una sensible apreciación de los complejos significados simbólicos y sociopolíticos de la más importante actividad artesana incaica.

Son limitadas las informaciones arqueológicas actualmente disponibles. La mayoría de ellas provienen de un conjunto de edificios en las ruinas de la capital provincial incaica, a menudo conocida como Huanuco Viejo, localizada en Huánuco Pampa. Este antiguo centro, que abarca más de $2.5 \mathrm{~km}^{2}$ y está a una altura de $4,000 \mathrm{~m}$, se encuentra a $150 \mathrm{~km}$ al noroeste de la moderna ciudad de Huánuco. La función del conjunto como centro de producción se descubrió durante un programa de investigación cuyo objeto eventual era trazar mapas y obtener muestras por medio de la excavación en toda la ciudad. Las excavaciones en el conjunto se realizaron en octubre, noviembre y diciembre de 1971, y la temporada terminó en enero de 1972. Aun no se ha terminado de analizar los cientos de miles de fragmentos y otros artefactos excavados. Además, con la continuación de la investigación, es posible que se encuentre nuevas instalaciones de producción. Lo que se propone ahora es una conclusión preliminar, que estará sujeta a modificaciones sustanciales mientras progrese el estudio.

En particular, la mayoría de las preguntas tecnológicas, tendrán que esperar análisis más detallados 5 . Lo que más resalta actualmente del estudio es la capacidad de por lo menos ciertos sectores de la economía incaica de producir bienes en cantidades relativamente grandes para un sistema preindustrial. Sin embargo, poco a poco nos estamos acercando a solucionar varios de los problemas relacionados con la organización de la producción artesanal y su papel en la economía y en la sociedad.

5 Aunque no se han estudiado los implementos encontrados durante nuestro trabajo más reciente, hay una larga tradición de interés en los aspectos técnicos del hilado y del tejido en los Andes. Entre las fuentes que tratan del tejido andino se incluyen: Bird (1954); Gayton (1967); O’Neale (1949).

\section{SUGERENCIAS RELATIVAS A LA} ORGANIZACIÓN DE LA PRODUCCIÓN

En general, hemos estado manejando tres ejes de organización que parecen ser especialmente importantes en el caso incaico, pero que corresponden sin duda también a otras sociedades complejas. En primer lugar, se considera hasta qué punto el estado controlaba la producción; en segundo lugar, el grado de especialización de los productores y, en tercer lugar, el tamaño y los principios de organización de la unidad productora. Estos dos últimos, como se verá más adelante, tienden a estar bastante relacionados en el caso de los incas.

La evaluación del papel del estado en la producción plantea un problema comparativo que precisa información sobre las actividades de producción organizadas por el propio estado incaico y aquellas organizadas por las diversas unidades socio políticas en niveles más bajos de la estructura. Las fuentes documentales, frecuentemente confunden el nivel estatal con organizaciones locales (los más bajos); pero proporcionan suficiente información para sugerir que la producción artesanal se llevaba a cabo en varios niveles. Algunos productos, especialmente el oro, la plata y ciertos tejidos parecen haber sido virtualmente monopolios del estado, el cual administraba tanto los materiales utilizados, como la producción misma (Murra 1956: 189). La producción especializada artesanal, por otro lado, no parece haber estado bajo la dirección total del estado. Moore (1958: 56) afirma que los kurackuna o jefes regionales, tenían acceso a la mano de obra de los especialistas. En 1567, los jefes Lupaqa de las cercanías del Lago Titicaca podían obtener todavía los servicios de los tejedores. Por esta época ya se había manifestado la influencia de la autoridad europea, así como otras motivaciones nuevas; pero el derecho a estos servicios parece haberse originado en el período precolombino (Murra 1964: 438). Un análisis más detallado de los documentos de la región llevaría probablemente a una mayor comprensión de la producción no agrícola en el nivel local. 
El éxito de la investigación arqueológica sobre la producción controlada por el estado, en contraposición con la que estaba bajo la dirección de unidades étnicas, depende de la capacidad para diferenciar entre los niveles estatales y locales en los restos materiales. Los que hemos trabajado con materiales incaicos en la región de Huánuco quizás somos culpables de exagerar la facilidad con la cual se puede lograr generalmente dicha distinción. No sólo se diferenciaban de las otras variedades locales la cerámica y la arquitectura de tipo inca imperial en esta región sino que también es distinta la distribución y la ubicación de los asentamientos. En esencia, hay una intrusiva red de poblados estatales que afectaban los patrones de población locales y preincaicos, una serie de pueblos que son dramáticamente diferentes tanto en su estilo como en sus funciones. Huánuco Pampa era parte prominente de esta red de poblaciones estatales, el contraste entre los pueblos locales y los estatales, y probablemente entre las actividades locales y aquellas relacionadas con el estado, tal vez no sea tan evidente en otros ámbitos del Tawantinsuyu, aunque el trabajo de Menzel (1959) en la costa sur del Perú sugiere que el caso podría haber sido algo parecido en partes de esa área. Naturalmente, esta idea no se puede aplicar a la región inmediata al Cuzco; es dudoso que la distinción entre lo estatal y lo regional sea pertinente en esta región, la cual como ha sugerido Rowe (1967), estaba altamente influida por la planificación estatal y probablemente se encontraba bajo mayor control directo del estado que las regiones muy remotas.

Teóricamente, debería ser posible estudiar la producción no agrícola no solamente en un contexto estatal, identificando principalmente a qué unidades pertenecían las instalaciones de producción y las residencias de los trabajadores. El problema más grave frente a la comparación entre la producción estatal y la no estatal es la dificultad de identificar cuáles eran las actividades productivas controladas indirectamente por el estado. Sabemos que había alguna producción para el estado en poblaciones que en realidad no eran del imperio y que parte de esta producción era probablemente administrada por la autoridad local. Refiriéndose a los Chupaychu, Ortiz de Zúñiga (1967: 239) nos informa que:

"la ropa de cunbi la hacían en su tierra que, mandó el ynga le pedía llevaban al Cuzco y otra parte donde les señalaba".

Con la evidencia disponible sólo podemos adivinar el monto de dicha producción, pero es probable que fuese sustancial.

Independientemente de la cantidad de bienes producidos para el estado incaico fuera de su propia red de poblaciones, la evidencia en Huánuco Pampa muestra ahora que ésta estaba directamente comprometida en la producción artesanal en una escala significativa. El material nuevo viene del área que se ha calificado como Conjunto 5 en la Zona VB, contigua a la plaza principal de la ciudad norte (véase figuras 1 y 2). Este conjunto de edificios ha sido llamado frecuentemente "el cuartel" (Harth-Terré 1964: 13-14). Uno de los aspectos más notables de este conjunto es el marcado grado de orden y de semejanza arquitectónica entre los edificios, que contrasta con gran parte de la ciudad. Esto sólo se explica por medio de una planificación explícita. Además, un examen cuidadoso del muro que rodea los edificios muestra que había acceso sólo en un punto, es decir, a través del Edificio b1.

Por medio de la excavación de una muestra de 20 de los 50 edificios del conjunto, se estableció que la producción no agrícola era una de las actividades principales. Mientras que varios productos podrían haber sido elaborados dentro del recinto, actualmente tenemos evidencia sólo de uno: el tejido. En una edición preliminar de este informe ${ }^{6}$ basada en evidencias muy iniciales, se sugirió que en el conjunto también se fabricaba cerámica. Una breve inspección de parte del material, así como trabajo adicional en el Conjunto VB5, junto con el volumen,

6 C. Morris y J. I. Santillana. "Nuevas evidencias sobre producción no-agrícola en el Estado Inka”. Ponencia presentada al "I Congreso Peruano del Hombre y la Cultura Andina", Lima, 1972. 


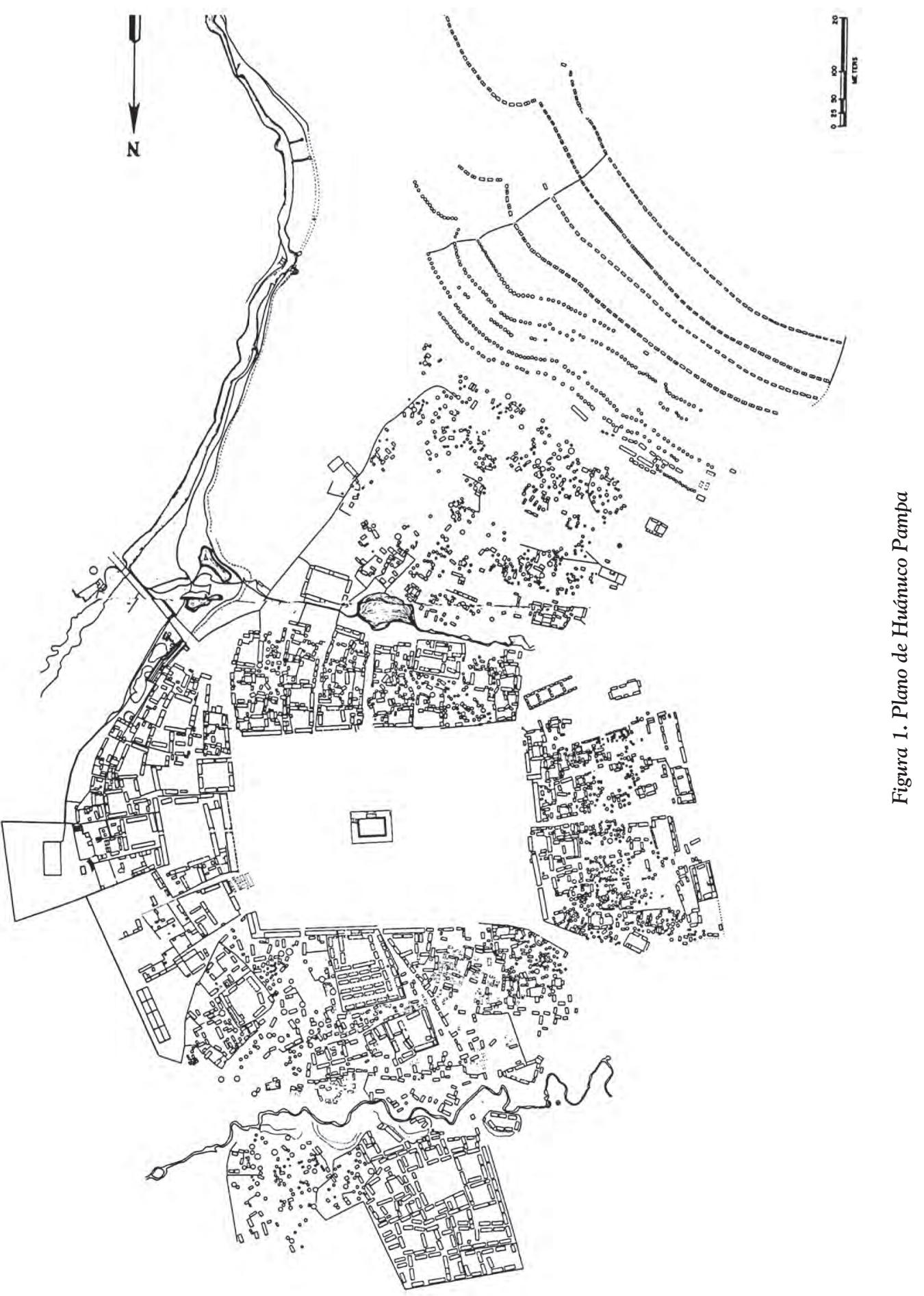




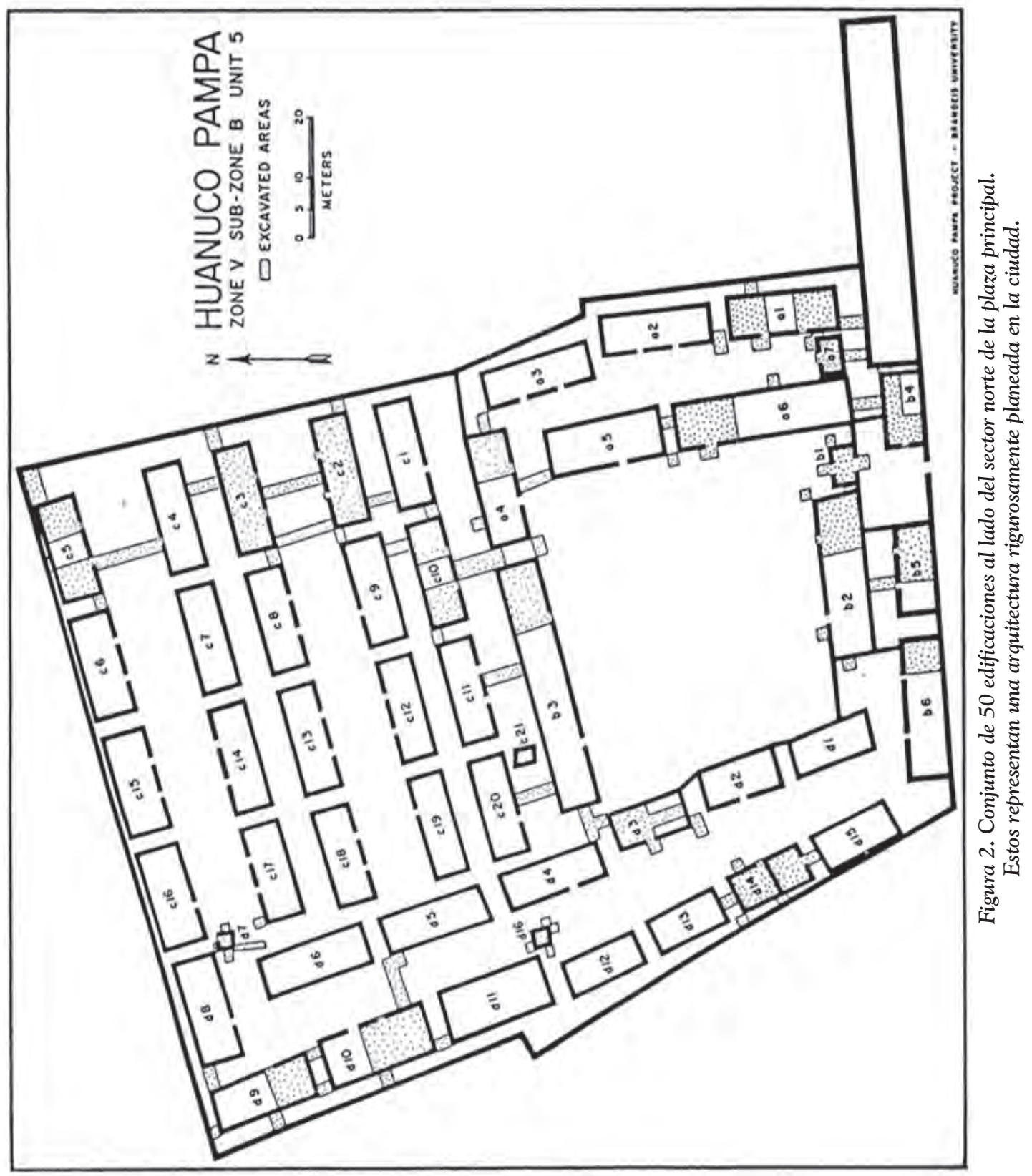


sustancialmente mayor de datos comparativos sobre las otras secciones de la ciudad, indican que si se producía cerámica dentro del recinto, era en muy pequeña escala. Por tanto, la única posibilidad de horno parece ser un fogón, que luego se usó como basurero. Recientemente se ha encontrado en otras partes del sitio numerosas muestras de lo que se pensaba que era moldes para bases de jarras. Ahora es evidente que eran soportes de jarras, relacionados con el uso de las vasijas y no con su fabricación. Pero si no se fabricaban ni se almacenaban allí, todavía está por aclarar la presencia de miles de jarras de diversas formas. La densidad de la cerámica en el Conjunto VB5 es varias veces mayor que la encontrada en otras áreas típicamente domésticas. La explicación de esto tendría que esperar un estudio sistemático de las colecciones, así como comparaciones adicionales con el resto de la ciudad. Sería lógico suponer que se utilizaban estas grandes cantidades de cerámica en actividades de producción, tal vez para teñir los tejidos. Otra hipótesis, analizada más adelante, sostiene que la cerámica se usaba posiblemente para la fabricación en gran escala de la chicha.

Como el tejido no se conserva en las tierras altas y húmedas de la sierra, las únicas pruebas que tenemos de su manufactura vienen de las herramientas usadas, y eso que estas estaban hechas con materiales de fácil deterioro, especialmente madera. El artefacto más numeroso era el huso, empleado como peso al hilar. Se ha hallado por lo menos 200 a 300 de éstos en el Conjunto VB5. La mayoría eran de cerámica, pero había algunos más finos de piedra pulida. Lo importante es que eran varias veces más comunes en este conjunto que en los otros edificios de Huánuco Pampa que supuestamente sirvieron principalmente como residencias. Las otras herramientas, más interesantes porque no son tan bien conocidas, eran de metal o hueso. Había un gran número de artefactos de hueso que casi se puede aseverar que sean los huesos pulidos con puntas muy agudas descritas por Cabo (Libro 14, capítulo XI; tomo II: 259), y que servían para controlar la tirantez de la tela durante su fabricación. También eran comunes otros artefactos rectangulares y con muestras de haber sido usados, aparentemente, para esparcir los hilos en el telar; agujas también abundaban. Se encontraron más de 100 artefactos relacionados con el hilado y con el acabado de la tela. La identificación inicial proviene de dos tejedores andinos, integrantes del equipo de excavación, quienes examinaron los objetos en el campo. Se necesitará estudios especializados y mayor información etnográfica para llegar a identificaciones más precisas, verificaciones de la función de los objetos, y para dar una idea más clara de los tipos de tejido que se producían.

La evidencia preliminar indica que las actividades en el Conjunto VB5 no se limitaban sólo a la producción. Antes de completar los análisis detallados de la investigación, es difícil distinguir entre los basureros y los fogones que eran el resultado de las actividades cotidianas y de la cocina, y los lugares que tal vez se asociaban con ciertos aspectos de la producción. Las cantidades de hueso sin tallar en el Conjunto VB5 son menores que en las áreas que se suponían de uso residencial. La evidencia actual apoya la teoría de algún tipo de vida cotidiana dentro del recinto. Sin embargo, es evidente que los patrones residenciales del conjunto se diferenciaban bastante de aquellos del resto de la ciudad. Si bien la zona no ha resultado ser un cuartel en el sentido militar, la impresión arquitectónica inicial probablemente es una interpretación fiel del estilo de las áreas residenciales. El hecho de que los trabajadores vivieran en el conjunto donde trabajaban, así como la disposición de su espacio residencial, son datos cruciales para comprender su organización.

No es posible en este momento expresar en términos cuantitativos precisos la importancia de la instalación de producción en Huánuco Pampa. Por la evidencia, estamos razonablemente seguros que todos los edificios casi idénticos (aproximadamente de 18 por 5 metros cada uno) que se encuentran arreglados en filas ordenadas, servían como de talleres-residencias (véase figura 2). Esto significa que había 40 edificios con un espacio interior total de más o me- 
nos 3,000 $\mathrm{m}^{2}$. Además, otros dos edificios, el b2 y el b3, contenían una gran cantidad de huesos y otras herramientas, que probablemente también e usaban para la producción y para actividades relacionadas con ella. Su arquitectura, en particular, indica que eran bastante distintos de los otros 40 edificios recién mencionados. Se propone que parte de esta diferencia radica en el hecho de que no se usaban como dormitorios.

Partiendo de la base de las 40 residenciastalleres, es difícil llegar a una indicación del número de personas que vivía en el conjunto. Toda la evidencia disponible da a entender que los edificios se usaron en forma contemporánea $\mathrm{y}$ es probable que todos fueran utilizados de manera intensiva durante la época posterior a la conquista española, cuando se interrumpió la función de la ciudad. No se conoce ninguna evidencia histórica que ayude directamente a calcular el tamaño del grupo de trabajadores; pero se supone que por lo menos 100 personas vivían y trabajaban en el conjunto. Esto implicaría un promedio de solamente 2.5 personas por cada edificio, sin duda un número mínimo si se tiene en cuenta la cantidad de material encontrado en la zona. El doble de este número sería probablemente más realista, pero parece casi imposible postular un total de más de 300 personas, cuando se considera la cantidad de espacio disponible y la proporción del mismo que debe haber sido ocupado por las vasijas de cerámica.

Cualquiera que sea el número exacto de productores o la cantidad precisa de su producción, se han de considerar grandes para una instalación de producción preindustrial. Además, la disposición arquitectónica indica que el control era estricto. Fuera del muro exterior del conjunto, las estructuras b1 y b4 parecen haber sido edificios administrativos de algún tipo No eran talleres ni residencias, y no estallamos exagerando si sugiriéramos que alojaban actividades relacionadas con los registros y con la seguridad.

A través de la evidencia de Huánuco Pampa podemos sostener con insistencia la gran importancia del estado y el grado de control directo en ciertas actividades de producción. Sería ven- tajoso disponer de mejores informaciones sobre los otros niveles de la economía y de la sociedad de la región para ver si esta instalación estatal, construida a fines del siglo XV o principios del XVI, tuvo alguna influencia notable sobre los patrones de producción a nivel local. iLas operaciones del estado implicaban acaso un aumento general en la producción de textiles y otros productos? Por otro lado, podría haber habido una centralización de varios de los aspectos de la producción, a expensas del rendimiento local. Quizás se trataba de una combinación de ambos factores. Sospechamos que lo que ocurría era que existían tendencias de cambia en la organización, las cuales colocaban bajo el control del estado la producción de cantidades cada vez mis grandes de ciertos bienes. Estudios más profundos de documentos regionales y de hallazgos arqueológicos quizás ayudarán a resolver estas preguntas tan cruciales para la comprensión de los patrones de cambio económico y político.

Ahora nos dirigimos hacia las cuestiones de la especialización por ocupaciones y del carácter de la unidad de producción. Aquí encontramos que la evidencia arqueológica indica que el Conjunto VBS era una instalación especializada, muy distinta a los otros grupos de edificios en la ciudad. No parece probable que la gente que trabajaba en aquella instalación se ocupara en ninguna otra actividad económica fuera del recinto. Debemos aguardar los estudios de los patrones de distribución de artefactos en el recinto antes de llegar a alguna conclusión final sobre el conjunto de actividades y sobre la subdivisión interna.

La evidencia disponible en este momento no sugiere un patrón de especialización interna rígido, y tiende a apoyar la sugerencia de que la mayoría de los miembros del grupo al que nos referimos practicaba labores semejantes, entre las que la producción de textiles era probablemente la primera actividad de una lista relativamente corta.

Se puede considerar mejor el concepto del grado de especialización como una parte del carácter y de la organización general de los grupos potenciales de trabajadores, campo en 
el cual los documentos proveen la mejor fuente. Esa información no se presenta en la forma de descripciones explícitas que se puedan aplicar directamente como interpretaciones, sino a manera de datos sobre el carácter general de la mano de obra disponible para el estado. La consideración de diversas opciones en un esfuerzo por contraponer la evidencia documental con la arqueológica, es típica del proceso interpretativo que le utiliza en muchos otros esfuerzos generales para reconstruir los patrones de actividad en la ciudad.

La etnohistoria sugiere que se pueden hacer distinciones entre los trabajadores del estado según varios criterios, incluida la labor desempeñada, la porción de tiempo dedicada al servicio del estado y el grado en que la comunidad local y las relaciones de parentesco eran alteradas por el cumplimiento de obligaciones hacia el estado. Es difícil definir de manera precisa las diversas categorías de trabajadores del estado, puesto que éstas eran relativamente flexibles y cambiaban rápidamente. Hay tres clases de trabajadores que son los que quizás ayudan más a entender la producción no agrícola; en Huánuco Pampa están relacionadas con el problema inmediato de interpretar la producción controlada por el estado: Son la Mit'a, compuesta por trabajadores reclutados en turnos; las aqllakuna, mujeres escogidas; y los mitmaqkuna, colonos. Todos se pueden considerar como patrones posibles, y habían sido utilizados por separado o conjuntamente por el estado incaico en las actividades de la producción. Sin embargo, es probable que solamente uno de ellos se aplique al complejo de producción tratado antes. Ciertamente que no hay divisiones que sugieran grandes distinciones de categorías o clase entre los trabajadores. Aquí solamente se puede bosquejar brevemente la naturaleza de estas tres clases. Para un tratamiento más completo de las diversas categorías de servicio al estado, véase Murra (1956: 264. 274) y Rowe (1946: 265-270).

a. La mit'a: Fuera de los nobles y los integrantes de algunas otras clases exoneradas de impuestos, todos los varones que gozaban de salud y fueran jefes de unidades domésticas estaban obligados a trabajar para el Inca sobre una base temporal y rotativa. Esta era la forma usual de trabajo en las tierras cultivadas del estado, en la construcción pública y en el ejército. En términos cuantitativos, es probable que la mit'a fuera la fuente más importante de mano de obra. Puesto que era un servicio temporal, la mit'a no alejaba a los conscriptos de sus contextos sociales y étnicos en las comunidades tradicionales. No conozco ningún vínculo definitivo entre la fuerza laboral de la mit'a y la producción textil u otro tipo de producción artesanal. Tendemos a asociar casi toda la artesanía con algún grado de especialización; sin embargo, el sistema de producción en gran escala que vemos en el Conjunto VB5 probablemente podía ser realizado por mano de obra temporal bajo la supervisión de especialistas. Hay la posibilidad adicional de que estos artesanos locales sirvieran al estado con una dedicación a tiempo parcial, cumpliendo con su obligación hacia la mit'a al desempeñar su trabajo habitual.

La razón primordial para dudar que el recinto de producción en Huánuco Pampa fuera administrado por trabajadores de la mit'a, es la indicación en las crónicas de que el hilado y una gran parte del tejido eran trabajos principalmente femeninos (Cobo [Libro 14, Capítulo XI]) 1956: Tomo II, 258; Guaman Poma [i1615?] 1936: 218-220; Rowe 1946: 24) aunque algunas fuentes mencionan la fina tela kumpi como producto del trabajo masculino (Cobo [Libro 14, Capítulo XI] 1956: Tomo II, 259), Además, la arqueología demuestra bastante específicamente la presencia de mujeres en el recinto. Encontramos más de una docena de alfileres de metal (T'upu) utilizados para sujetar la ropa femenina (véase los diversos dibujos de Guaman Poma, 1936: 300 por ejemplo) y no había artefactos que pudiéramos asociar con la presencia o con actividades masculinas, por lo menos en esta etapa de nuestro análisis.

b. Los mitmaqkuna: Los colonos mitmaq eran gentes trasladadas por el estado de un lugar a otro en grupos relativamente grandes, una 
forma de colonización dirigida por el estado. La razón que se da generalmente para explicar estos cambios de lugar de las poblaciones, es que los cambios apoyaban el mantenimiento de la seguridad interna. Sin embargo, Cieza (1959:60-62) sugiere que también tenían funciones económicas. Si bien el tamaño exacto de las unidades mitmaq no está claro y probablemente variaba mucho, parece cierto que eran grupos de varias familias, frecuentemente comunidades enteras.

Cieza sostiene que los mitmaqkuna eran a veces artesanos, plateros, picapedreros, y menciona específicamente a los tejedores. En uno de los edificios del Conjunto VB5 se hallaron algunos fragmentos al estilo de la cerámica negra de Chimú, hechos con materiales de la región, lo cual llevaría a pensar en la posibilidad de que sus ocupantes fueran mitmaqkuna del Chimú. Pero como los fragmentos constituían un porcentaje insignificante de la colección del edificio y como parecen haber faltado en las otras estructuras, su presencia no apoya fuertemente la posibilidad mencionada. La arquitectura, por otro lado, parece contradecir la idea de los mitmaqkuna. Presuntamente, los mitmaqkuna se caracterizaban como un grupo de unidades familiares interrelacionadas. Una distribución, con edificios casi idénticos en calles relativamente angostas, parece más adaptable a su utilización por individuos, en vez de familias. Si bien tenemos que admitir que la arquitectura en sí misma no es completamente convincente, nuestra supuesta expectativa para una colonia de mitmaqkuna sería un grupo de pequeños recintos, cada uno con sitio para un patio y algún grado de independencia para la unidad familiar (véase Rowe 1944 y Morris 1971 sobre la arquitectura incaica).

c. Las aqllakuna: Las aqllakuna han sido usualmente vinculadas con la religión (Rowe 1946: 269.; Cobo [Libro XII, Capítulo XIX] 1956, II, 98), pero el material documental también sugiere que era un grupo variado y que sus servicios incluían actividades que no eran específicamente religiosas (Rowe 1946: 269; Guamán Poma [1615] 1936: 298-299). las aqllakuna eran solteras y, en general, jóvenes.
A menos que un análisis futuro cambie nuestra interpretación de los restos materiales en Huánuco, lo más probable parece ser que los ocupantes del Conjunto VB5 fueron algún tipo de aqllakuna, o por lo menos un grupo con una estructura semejante.

Las mujeres y las actividades femeninas se asociaban casi por completo con el recinto; en términos arquitectónicos, la unidad de residencia era probablemente una unidad no familiar; el control estricto de acceso al recinto demuestra medidas de seguridad que no tienen comparación en la ciudad, y estas parecerían justificables sólo si los ocupantes del recinto pertenecieran a alguna clase social especial, la cual requiriera una seguridad excepcional. Estas características describen mejor a las mujeres escogidas que a cualquier otro grupo que pudiera haber estado presente en un centro administrativo incaico. No obstante, no hay evidencia de los cargos religiosos que se supone que también desempeñaban.

En una comunicación personal el Dr. John H. Rowe ha sugerido que la evidencia arqueológica, fuera examinada en términos de la posibilidad de que allí se produjera la chicha, puesto que se sabe que las aqllakuna la hacían (Rowe 1964: 269; Guaman Poma [1615] 1936: 299). Quizás la mejor hipótesis actualmente disponible para explicar las grandes cantidades de cerámica en el recinto es que fuera utilizada en la elaboración de Chicha. Cobo ([Libro XIV) Capítulo IV] 1956; II: 242) nos informa en la preparación y el consumo de la chicha se utilizaban numerosas vasijas. Durante la temporada de excavaciones de 1972, trabajamos en otra área abundante en cerámica (en el sector oriental de la ciudad) que se ha interpretado provisionalmente como un lugar donde se elaboraba la chicha y otros productos alimenticios para el consumo general (Morris mss, Stein, 1975). Al comparar las formas de las jarras y los restos botánicos encontrados en éstas y otras colecciones dispondremos de algunas pruebas excelentes sobre la posible producción de la chicha.

No es necesario aceptar literalmente la clasificación compleja de las mujeres escogidas 
indicada por Guamán Poma ([1615] 1936: 301 302) para suponer que, además de la diferencia en categorías de edad, había distinciones importantes en las actividades económicas realizadas por varios grupos de aqllakuna. En realidad, al considerar estas variaciones, parece probable que hubiera más de una casa para las mujeres escogidas en un centro importante como Huánuco Pampa; además, el Conjunto VB5 puede ser solamente un recinto ocupado por mujeres cuyas responsabilidades económicas sobrepasaban casi completamente cualquier cargo o función religiosa que tuvieran.

La manera por la cual las sociedades complejas movilizan y organizan una fuerza laboral para realizar ciertas tareas esenciales representa una de sus dimensiones más importantes. En el caso de sociedades complejas tempranas, parece muy probable que grupos estructurados según líneas religiosas, y a veces de parentesco, se dirigieran gradualmente hacia funciones económicas, políticas y militares bajo el servicio de una autoridad centralizada (Murra 1956, Cap. VIII y 1962a: 722; Adams 1956). Un factor importante para, el desarrollo de los estados antiguos era la capacidad para manipular las instituciones tradicionales y las unidades de organización de tal forma que pudieran servir a distintas funciones. Tenemos evidencia, entre los incas, de una mano de obra considerable, controlada directamente por el estado. Si las investigaciones posteriores confirmaran nuestra identificación de esa fuerza como un grupo de mujeres escogidas, podremos empezar a ver la interacción de la complicada red de factores económicos, políticos y religiosos que en traban en la formación y en el mantenimiento de estos grupos.

\section{EL CONTEXTO INSTITUCIONAL DE}

\section{LA PRODUCCIÓN CENTRALIZADA}

Por nuestro conocimiento general de las sociedades urbanas antiguas hemos llegado a considerar que la producción especializada no agrícola es un rasgo importante. En consecuencia, no nos sorprende encontrar evidencia sustancial de esta producción en el caso de la población incaica, Mientras que algo de especialización en los bienes manufacturados es una característica general de toda civilización antigua y probablemente siempre tenía algo que ver en sus procesos de desarrollo, la especialización no siempre encajaba dentro del mismo contexto socioeconómico, ni desempeñaba el mismo papel.

Obviamente, el panorama de la organización económica incaica es todavía incompleto. Sin embargo, hay dos aspectos generales del sistema económico que pueden ayudar a explicar por qué era importante para el estado incaico en expansión disponer de un gran control directo sobre la producción, cómo se realizaba ese control estatal y por qué se daba énfasis a productos tales como el tejido. El primero de estos rasgos cruciales es que los incas ponían énfasis en las relaciones de intercambio recíproco, antes que en el trueque o el uso de mercados (Murra 1956: Cap. VI). Ligado a esto vemos que el estado dependía del trabajo humano para muchos bienes, en vez de tener como al base de renta el sistema de tributos en especie. El segundo aspecto es la gran capacidad de los dirigentes a varios niveles de la sociedad para trasladar a la población de sitio en sitio, colocando a la gente donde tuviera acceso a los recursos esenciales. En la mayoría de los casos, estos recursos eran naturales, y tenían que ver con la colocación de pueblos en diferentes zonas ecológicas (Murra 1967; 1968; 1972; 1975). Pero cuando se llega al nivel de organización estatal, se trataba frecuentemente del traslado de la gente a situaciones esencialmente urbanas, destinándola no sólo al control de recursos naturales, sino a la organización o supervisión de la población laboral.

La práctica de la reubicación de la población y del traslado de gente para lograr diferentes fines, era común a los tres grupos de trabajadores estatales tratados antes. En el caso de la producción artesanal, facilitaban la centralización de la producción, las rentas basadas en la energía humana en combinación con la capacidad de reubicar a la gente. Si se podía traer productores en número suficiente a los centros 
de la administración estatal, se simplificaba el control directo. Si nuestra interpretación actual de Huánuco Pampa es acertada, éste era tal tipo de centro. Había pocos aspectos de la vida de la ciudad en que el estado no dominara. La falta de casi toda influencia local en la arquitectura y en los artefactos señala la posibilidad de un control estatal estricto de la vida de la ciudad, y no hace pensar que hubiera interacción libre e informal con las regiones remotas. Además, los ocupantes del Conjunto VB5 no eran los únicos residentes de la ciudad que estaban ahí como resultado de procedimientos estatales relativos a la renta y a la colonización. Probablemente la mayoría de su población, si no toda, era reclutada a través de similares mecanismos (Morris 1972b).

Regresando ahora a la importancia del intercambio recíproco, es necesario los métodos por los cuales el estado se deshacía de sus productos. La distribución de bienes tiene que ver, por su lado, con las relaciones recíprocas entre el estado y aquellos que trabajaban para él. El tipo de interacción varía según la clase o el trabajador, pero parece que el estado tenía que organizar el traslado y proveer hospitalidad en todos los casos (Murra 1956: 169; 1958). Nuestro trabajo arqueológico en Huánuco Pampa ha indicado que el estado hacía muchos esfuerzos para cumplir con sus obligaciones de abastecimiento y hospitalidad (Morris 1972b; y mss).

El material documental nos dice que se esperaba que el estado proporcionara los alimentos y otras necesidades que podían tener aquellos que lo servían. La arqueología muestra que para lograr este fin, se constituyeron 497 almacenes en Huánuco Pampa. La mayoría de estos se utilizaban para almacenar alimentos, probablemente dirigidos en gran parte a asegurar una provisión estable de comida para la ciudad misma (Morris 1967). La arqueología sugiere también que el estado suministraba las vasijas en las cuales preparaban, guardaban y servían los alimentos (Morris y Thompson 1970). Aunque ahora no parece que producía cerámica en el Conjunto VB5, la uniformidad estilística y material de los fragmentos es tal que apoya fuertemente la idea de que la producción de la cerámica estaba centralizada y que probablemente se producía cerca de Huánuco Pampa o en la ciudad misma. De todos los productos alimenticios suministrados, la chicha era quizás el más importante. Ciertamente, ésta resultaba un elemento esencial en un centro donde el trabajo del estado se llevaba a cabo a menudo dentro de un contexto ceremonial (Murra 1958; 1960). Además de la posibilidad de su producción en el Conjunto VD5, creo que podemos demostrar lo siguiente: que la chicha se preparaba para varios fines distintos en las diferentes partes de la ciudad, que era un producto principal esencial en la economía de un centro administrativo y que hacer chicha constituía una actividad de tanta importancia como tejer.

El tejido era particularmente vital en la reciprocidad. En los Andes se utilizaba en casi todas las relaciones sociales, políticas y económicas de importancia (Murra 1962a). Bienes como la chicha o la cerámica eran probablemente artículos de abastecimiento urbano y con pocas excepciones se consumían o se utilizaban cerca de sus lugares de producción. Sin embargo, el tejido pesaba menos y tenía más valor, por eso, hay que considerar su producción en el contexto de un área y un ambiente político-económico mucho más extenso. La evidencia arqueológica disponible nunca ha podido rastrear en la sierra las distribuciones de textiles perecederos. Pero la importancia concedida a los tejidos en los documentos implica seguramente que se redistribuían más allá de la ciudad a manera de regalos o de reciprocidad. Usualmente, las relaciones económicas y políticas entre el Inca y un grupo nuevamente sojuzgado se iniciaban mediante la entrega de tejidos (Murra 1962a: 721). Nuestro trabajo arqueológico en Ichu, el pueblo donde vivió el jefe de uno de los grupos locales antes, durante y después de la época incaica (Ortiz [1562] 1967: 55-60) mostró que una gran cantidad de la cerámica asociada con Huánuco Pampa había llegado allí de alguna manera (Thompson 1967: 360). Este es uno de los muy raros casos en que se ha encontrado en un pue- 
blo remoto de la sierra la cerámica producida por o para el estado, y muestra el flujo de ciertos bienes a lo largo de líneas de poder político.

Para los procesos económicos y políticos del estado incaico, era esencial una gran variedad de bienes agrícolas. Algunos eran importantes porque apoyaban intrincadas operaciones administrativas y militares; otros, como el tejido, circulaban en términos redistributivos más amplios, extendiendo los artículos del Inca y los símbolos de su poder hasta el campo. La disponibilidad de estos productos afectaba directamente lo que podía hacer el estado y lo que no podía hacer. Los aumentos en la producción de tejido y de otros bienes, algunos de los cuales no conocemos todavía, permitían al estado emprender nuevos vínculos económicos y políticos, renovando y reforzando continuamente los que ya tenía. La producción estatal, o por lo malos el acceso extensivo del estado al tejido y a otros productos especiales, alimentaba una economía y una política en expansión. Es en este contexto de rápido crecimiento económico y político, que se daba principalmente obedeciendo a una serie de principios recíprocos peculiares y no bien entendidos todavía, en el que se explica el establecimiento por el estado de un complejo de producción en Huánuco Pampa, rigurosamente planificado y controlado.

El análisis del desarrollo económico y político de cualquier sociedad requiere datos sólidos sobre muchos aspectos de la producción, de la organización y del control. Para el estudio de sociedades contemporáneas se obtiene esta información por medio de estadísticas cuidadosamente recopiladas, junto con materiales detallados sobre los contextos de los cuales se extrajeron. En el caso de los incas no contamos con este tipo de fuente, fuera de los pocos casos en los cuales se transcribió kiphu, razón por la que dependemos de las reconstrucciones derivadas en gran parte de la documentación y la arqueología. Los datos obtenidos de esta manera son difíciles e ineficaces de usar cuando se contrastan con aquellos disponibles para los estudiosos de sociedades modernas. No obstante, las investigaciones diseñadas cuidadosamente imaginativamente para aprovechar los diversos tipos de materiales disponibles, pueden ofrecer una nueva y útil comprensión, aún en las difíciles cuestiones de organización e institucionales.

Deseo subrayar un último punto referente al estudio del desarrollo económico y político en los Andes y en otros lugares. Aunque se ha reconocido, por lo menos desde Childe (1950), la importancia de los bienes no agrícolas en la emergencia de las sociedades complejas, ha habido una tendencia, sobre todo en la arqueología, a dirigir las investigaciones a cuestiones de productos de subsistencia. Sin sugerir que disminuyamos el énfasis en los estudios de la economía de subsistencia, considero que necesitamos dedicar mucho más esfuerzo a la investigación de aquellos bienes no agrícolas (por ejemplo el tejido en el caso andino) que tenían un valor y significado mucho más allá de su simple utilidad económica. Son estos los productos no agrícolas que nos dan acceso al conocimiento de algunas de las dimensiones sociales y políticas, tanto como económicas, de una sociedad. Su penetración en múltiples esferas es lo que les da una función clave en la emergencia de formas centralizadas de organización y en la determinación de la estructura institucional de la sociedad.

\section{Agradecimientos}

Nuestro trabajo fue subvencionado por la $\mathrm{Na}$ tional Science Foundation (GS 28815); y autorizado por las Resoluciones Supremas Nos. 015 y 1038 (1972). Además, deseo agradecer al personal del Proyecto Arqueológico en Huánuco Pampa y a las numerosas personas cuya ayuda ha sido inapreciable. Van agradecimientos especiales al Arqueólogo Idilio Santillana V., entonces estudiante de la Universidad de San Cristóbal de Huamanga, que dirigió las excavaciones en el Conjunto VB5 y que preparó la versión preliminar de la figura 2. También estoy agradecido al Sr. Delfín Zúñiga y a la Sra. Jean Mohan, quienes colaboraron en la última versión de la figura 2 . 
Finalmente, estamos en deuda con John V. Murra, Eva Hunt, John H. Rowe, George Cowgil y Franklin Pease por valiosas observaciones y críticas al borrador de este informe.

\section{BiblografíA}

Adams, Robert Mc.

1956 "Some hypotheses on the development of early civilizations". American Antiquity, 21: 227-232.

1965 Land behind Baghad. University of Chicago Press, Chicago.

1966 The evolution of urban society. Aldine, Chicago.

Adams, Robert Mc. and Hans J. Nissen

1972 The Urok countryside: the natural setting of urban societies. University of Chicago Press, Chicago and London.

Bird, Junius

1954 Paracas fabrics and Nazca needlework. Textile Museum. Washington, D..C.

Calnek, Edward E.

1972 "Settlement pattern and chinampa agriculture at Tenochtitlan". American Antiquity, 37(1): 104.115.

Charlton, Thomas H.

1969 "Ethnohistory and archaeology: postconquest Aztec sites". American Antiquity, 34(3): 286-294.

Childe, V. Gordon

1950 "The urban revolution". Town Planning Review, 21: 3-17.

Cieza de León, Pedro

[1550] 1959 The Incas of Pedro de Cieza de León. Translated by Harriet de Onis and edited by Victor W. von Hagen. University of Oklahoma Press, Norman.

Cobo, Bernabe

[1653] 1964 Historia del nuevo mundo. Biblioteca de Autores Españoles, Vols, 91-92. Madrid.
Gayton, Anna Hadwich

1967 "Textiles from Hacha”. In Nawpa Pacha 5: 1-13.

Harth Terre, Emilio

1964 "El pueblo de Huánuco Viejo". El Arquitecto Peruano, 320 (21): 1-20.

Jacobs, Jane

1962 The economy of cities. Random House, New York.

Menzel, Dorothy

1959 "The Inca ocupation of the south coast of Peru". South-western Journal of Anthropology, 15: 125-142.

Millon, Rene

1970 "Teotihuacan: completion of map of giant ancient city in the Valley of Mexico", Science 170: 1077-1082.

Moore, Sally Falk

1958 Power and proprerty in Inca Peru.Columbia University Press, New York.

Morris, Craig

1966 "El Tampu Real de Tunsucancha". Cuadernos de Investigación, Antropología 1: 95-107. Universidad Nacional Hermilio Valdizán, Huánuco.

1967 Storage in Tawantinsuyu. Doctoral dissertation, University of Chicago.

1971 "The identifcation of function in Inca architecture and ceramics". Proceedings of the 39th Congress of Americanists, Tomo XXVIII, 3: 135-144.

1972a "State settlements in Tawantinsuyu: a strategy of compulsory urbanism". Contemporary archeology: a guide to theory and contributions. Mark P. Leone, ed., pp. 393-401. Southern Illinois University Press, Carbondale (versión española en Revista del Museo Nacional, XXXIX 127-141, Lima [1973])

1972b El almacenaje en dos aldeas de los chupaychu. Ortiz de Zúñiga. Vol. II: 383-404.

Mss "Inca urbanism: new evidence from a provincial capital". Unpublished manuscript. 
Morris, Craig and Donald E. Thompson

1970 "Huánuco Viejo: an Inca administrative center”. American Antiquity, 35 (3): 344-362.

Murra, John V.

1956 The economic. Organization of the Inca state, Doctoral dissertation. University of Chicago. Traducción español. La organización económica del estado inca, Siglo XXI, Mexico 1978.

1958 "On Inca political structure". Systems of political control and bureaucracy in human societies. Vern F. Ray, ed., pp. 30-41. University of Washington Press, Seattle.

1960 "Ríte and crop in the Inca state". Culture in history, essays in honor of Paul Radin. Stanley Diamond, ed. Columbia University Press, New York.

[1958] 1962a "La función del tejido en varios contextos sociales del estado Inca". Actas del II Congreso Nacional de Historia del Perú, Lima. También en American Anthropologist, 64:710-728.

1962 b "An archeological "restudy" of an Andean ethnohistorical account", American Antiquity, 28: 1-4.

1964 "Una apreciación etnológica de la Visita”, García Diez de San Miguel, Visita hecha a provincia de Chuchito... en el año 1567. Documentos Regionales para la Etnología y Etnohistoria Andinas I. Casa de la Cultura del Perú, Lima.

1967 "La Visita de los Chupachu como fuente etnológica". Ortiz de Zúñiga [1562] (1967).

1968 “An Aymara kingdom in 1567”, Ethnohistory, 15: 115-151. "Current research and prospects in Andean ethnohis- tory", In Latin American Research Review V: 3-36.

1972 "El control vertical de un máximo de pisos ecológicos en la economía de las sociedades Andinas", Ortiz de Zúñiga [1562] (1972).

1975 Formaciones económicas y políticas del mundo andino, Instituto de Estudios Peruanos, Lima.

O’Neale, Lila M.

1949 "Weaving". Handbook of South American Indians, 5. Washington, D.C.

Ortiz de Zuñiga, Iñigo

[1562] Visita de la provincia de León de Huánuco en 1562, Vols. I y II.

1967-72 Documentos para la Historia y Etnología de Huánuco y la Selva Central, 1 y 2. Universidad Nacional Hermilio Valdizán, Huánuco.

Guaman Poma de Ayala, Felipe

[1615] 1936 Nueva coronica y buen gobierno. Institut d'Ethnologie, París.

Rowe, John H.

1944 "An introduction to the archaeology of Cuzco". Peabody Museum Papers $27(2)$.

1946 "Inca culture at the time oí the Spanish conquest". En Handbook of South American Indians 2: 185-330. Washington, D.C.

1967 "What kind of settlement was Inca Cuzco?". In Nawpa Pacha 5: 55-77.

Stein, Pat $\mathrm{H}$.

1975 "The Inca's hospitality: Food processing and distribution at Huánuco Viejo". Ponencia a la reunión anual; Society for American Archaeology, Dallas. 